\title{
Characterization of the Propagation Properties of the Half-Mode Substrate Integrated Waveguide
}

\author{
Qinghua Lai, Student Member, IEEE, Christophe Fumeaux, Senior Member, IEEE, \\ Wei Hong, Senior Member, IEEE, and Rüdiger Vahldieck, Fellow, IEEE
}

\begin{abstract}
The propagation properties of the half-mode substrate integrated waveguide (HMSIW) are studied theoretically and experimentally in this paper. Two equivalent models of the HMSIW are introduced. With the first model, equations are derived to approximate the field distribution inside and outside the HMSIW. Using the second model, an approximate closed-form expression is deduced for calculating the equivalent width of an HMSIW that takes into account the effect of the fringing fields. The obtained design formulas are validated by simulations and experiments. Furthermore, the attenuation characteristics of the HMSIW are studied using the multiline method in the frequency range of 20-60 GHz. A numerical investigation is carried out to distinguish between the contributions of the conductive, dielectric, and radiation losses. As a validation, the measured attenuation constant of a fabricated HMSIW prototype is presented and compared with that of a microstrip (MS) line and a substrate integrated waveguide (SIW). The SIW is designed with the same cutoff frequency and fabricated on the same substrate as the HMSIW. The experimental results show that the HMSIW can be less lossy than the MS line and the SIW at frequencies above 40 GHz.
\end{abstract}

Index Terms-Half-mode substrate integrated waveguide (HMSIW), propagation properties.

\section{INTRODUCTION}

$\mathbf{R}$ ECTANGULAR waveguides are widely used in many microwave and millimeter-wave systems because of their high performance in terms of quality factor and power-handling capacity. However, the integration of these 3-D devices with planar circuits is difficult due to their high profile. As a solution to this problem, some integrated waveguides, such as the substrate integrated waveguide (SIW) [1], post-wall waveguide [2], and laminated waveguide [3] have been proposed. While main-

Manuscript received December 22, 2008; revised April 21, 2009. First published July 17, 2009; current version published August 12, 2009. This work was supported by the National Natural Science Foundation of China (NSFC) under Grant 60621002 and by the Electromagnetic Fields and Microwave Electronics Laboratory (IFH), Switzerland.

Q. Lai is with the Laboratory for Electromagnetic Fields and Microwave Electronics, ETH Zürich, Zürich CH-8092, Switzerland, on leave from the State Key Laboratory of Millimeter Waves, School of Information Science and Engineering, Southeast University, Nanjing 210096, China (e-mail: qhlai@emfield.org).

W. Hong is with the State Key Laboratory of Millimeter Waves, School of Information Science and Engineering, Southeast University, Nanjing 210096, China (e-mail: weihong@seu.edu.cn).

C. Fumeaux is with the School of Electrical and Electronic Engineering, The University of Adelaide, Adelaide 5005, South Australia (e-mail: cfumeaux@eleceng.adelaide.edu.au).

R. Vahldieck is with the Laboratory for Electromagnetic Fields and Microwave Electronics, ETH Zürich, Zürich CH-8092, Switzerland (e-mail: vahldieck@ifh.ee.ethz.ch).

Digital Object Identifier 10.1109/TMTT.2009.2025429 taining the advantageous characteristics of conventional rectangular waveguides, these integrated waveguides present additional advantages of low profile and low cost, which confer them a prospect of wide application in integrated circuits. In fact, during the recent years, the propagation properties of the SIW have been studied extensively [4]-[7] and a number of high-performance planar components have been developed based on SIW structures [8]-[12]. However, for many practical applications, the SIW is still too wide. Aiming at a further reduction of the transverse size of the SIW, the concept and geometry of a folded substrate integrated waveguide (FSIW) was introduced in [13] and exploited to develop components such as filters [14]. Recently, approximate formulas were proposed for the FSIW design and for the calculation of its phase constant in [15]. Nevertheless, a close consideration of the structure of the FSIW indicates that its fabrication is rather complicated and costly since it consists of three metallic layers. Additionally, compared with the SIW, the total metallic surface area enclosing the fields (considering the top, middle, and bottom layers) is not reduced. As a result, the FSIW encounters ohmic losses at the same level as the SIW in the millimeter-wave band.

The concept of the half-mode substrate integrated waveguide (HMSIW) was proposed recently in [16] and its geometry is depicted in Fig. 1(a) and (b). It can be observed, on one hand, that both the waveguide width and the surface area of the metallic sheets are reduced by nearly half compared with the SIW, and on the other hand, that the fabrication complexity is maintained at the same level as for the SIW. Several components have been developed based on the HMSIW since its introduction [17]-[20]. However, as one of the most important aspects for a transmission line, the propagation properties of the HMSIW still have not been studied systematically.

The focus of this study includes the determination of the field distribution inside and outside the HMSIW, the calculation of the phase constant, and the characterization of the attenuation. In Section II, approximate equations for the field distribution and a set of explicit design formulas are derived according to two equivalent models of the HMSIW. Subsequently, in Section III, the conductive, dielectric, and radiation losses in the HMSIW are distinguished by utilizing a numerical analysis procedure. Finally, the attenuation of a fabricated HMSIW is measured and compared with that of both a SIW and a microstrip (MS) line in the frequency range of 20-60 GHz.

\section{Modal Analysis AND Design Equation}

For the investigation on the field modes and the propagation constant of the HMSIW, two prototypes are created in the configuration depicted in Fig. 1(a) and simulated using the simula- 


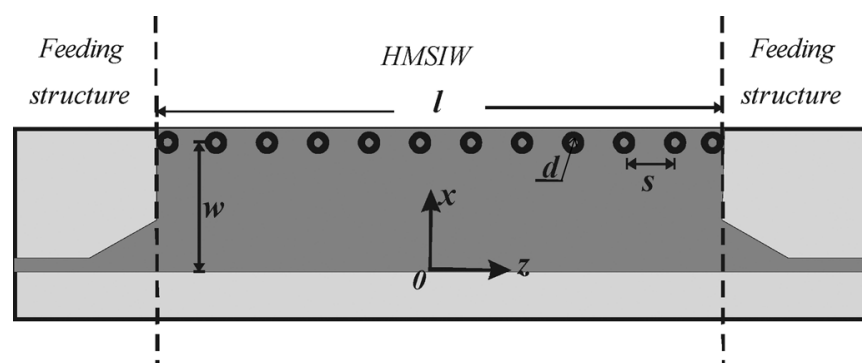

(a)

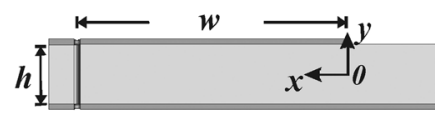

(b)

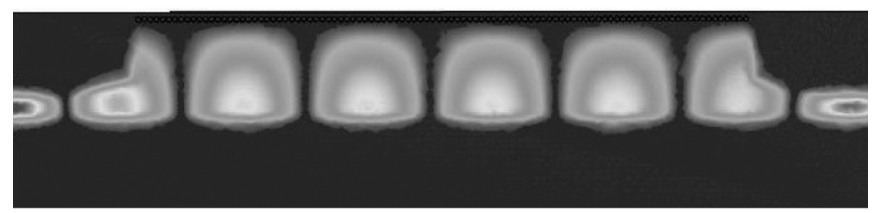

(c)

Fig. 1. (a) Top view of the configuration of an HMSIW fed by an MS line. The substrate is shown as light grey, and the dark grey areas are metallized. The metal posts (vias) are shown as circles. (b) Side view of the configuration of the HMSIW. (c) Instantaneous electric field distribution of the quasi- $\mathrm{TE}_{0.5,0}$ mode in the $H$-plane at $10 \mathrm{GHz}$.

tion tools Ansoft High Frequency Structure Simulator (HFSS) and CST Microwave Studio. The first prototype is designed for operation in the $X$-band and has a width of $w=10 \mathrm{~mm}$ and a height of $h=0.508 \mathrm{~mm}$. The second HMSIW prototype is designed for the frequency range of $20-60 \mathrm{GHz}$ and has a width of $w=2.5 \mathrm{~mm}$ and a height of $h=0.254 \mathrm{~mm}$. Both prototypes are built on a Duroid substrate with a permittivity of 2.2 and a loss tangent of 0.001 . The vias in both prototypes share a common diameter $d=0.5 \mathrm{~mm}$, as well as a spacing $s=0.6 \mathrm{~mm}$.

Due to the large ratio of HMSIW width to height and the discrete arrangement of the metallic vias, only the quasi- $\mathrm{TE}_{p-0.5,0}$ $(p=1,2, \ldots)$ modes can propagate in the HMSIW. The $H$-plane electric field distribution corresponding to the fundamental quasi- $\mathrm{TE}_{0.5,0}$ mode is plotted in Fig. 1(c). It can be observed that the fundamental mode in the HMSIW is quite similar to half of the dominant quasi- $\mathrm{TE}_{10}$ mode in the SIW. This explains the mode nomenclature with index 0.5 and the origin of the denomination for the half-mode SIW.

\section{A. First Equivalent Model: Field Distribution in the HMSIW}

Simulating the HMSIW prototypes in CST yields the distribution of the normalized electric field component $E_{y}$ in cross section, as illustrated in Fig. 2. The plot shows that the $y$-component of the electric field can be approximated as a quarter-cosinusoid in the HMSIW $(0<x / w<1)$ and decays rapidly beyond the open side of the waveguide $(x / w<0)$.

A first equivalent model, as shown in Fig. 3, is proposed for the analytical determination of the fields inside and outside the HMSIW. This model is composed of a leaky-wave guide in a homogeneous medium with a permittivity of $\varepsilon_{0, \text { eff }}$. The leaky-wave guide shares a common structure and dielectric material with the original HMSIW, whereas the homogenous surrounding medium is used to equivalently represent the effects of

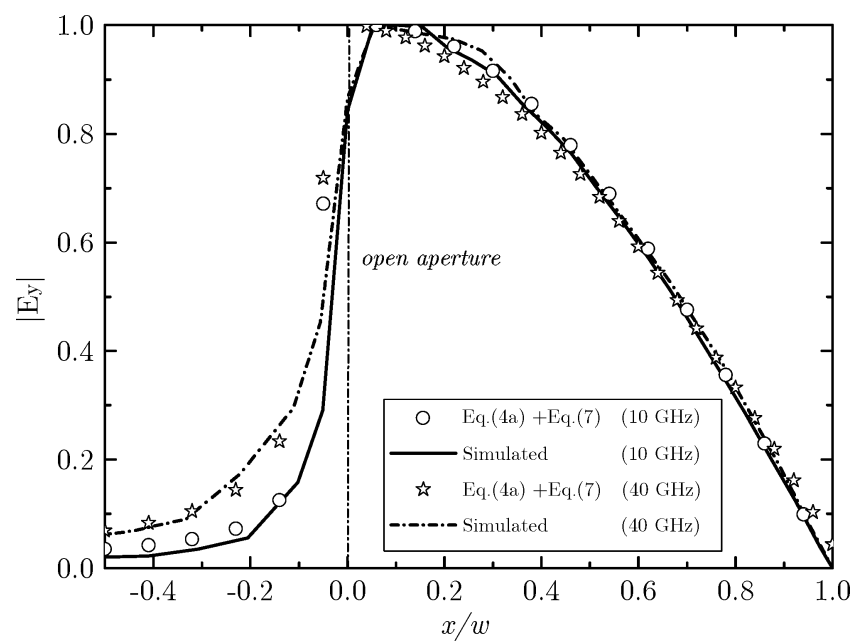

Fig. 2. Normalized electric field in cross section for two HMSIW prototypes operating in $X$ - and $K a$-band, respectively.

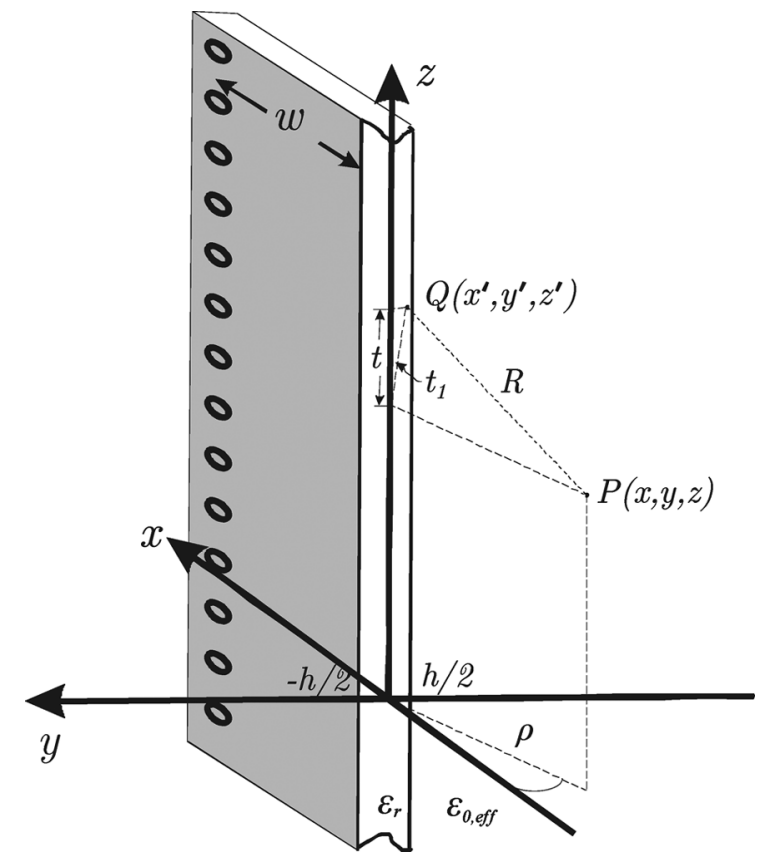

Fig. 3. First equivalent model of the HMSIW and the corresponding coordinates for the calculation of the field distribution.

the substrate, ground plane, and free space outside the HMSIW. The value of $\varepsilon_{0, \text { eff }}$ lies, therefore, in between the permittivity of free space $\varepsilon_{0}$ and that of the substrate material $\varepsilon_{r}$. For an HMSIW made of thin substrate extending past the open aperture, the value of $\varepsilon_{0 \text {,eff }}$ is close to $\varepsilon_{0}$. The fields in the leaky-wave guide and in the homogeneous medium correspond to those inside and outside the HMSIW, respectively.

According to the simulated electric field distribution inside the HMSIW $(0<x / w<1)$, as given in Fig. 2 , the function $\psi$ in the electric vector potential $\vec{F}=\vec{a}_{z} \psi$ is assumed in the following form for the quasi- $\mathrm{TE}_{p-0.5,0}(p=1,2, \ldots)$ modes

$$
\begin{aligned}
\psi_{p-0.5, q}= & A_{p-0.5, q} \cos \left[k_{x,(p-0.5, q)}(w-x)\right] \\
& \times \cos \left(k_{y,(p-0.5, q)} y\right) e^{-j k_{z,(p-0.5, q)}}, \quad 0 \leq x \leq w
\end{aligned}
$$




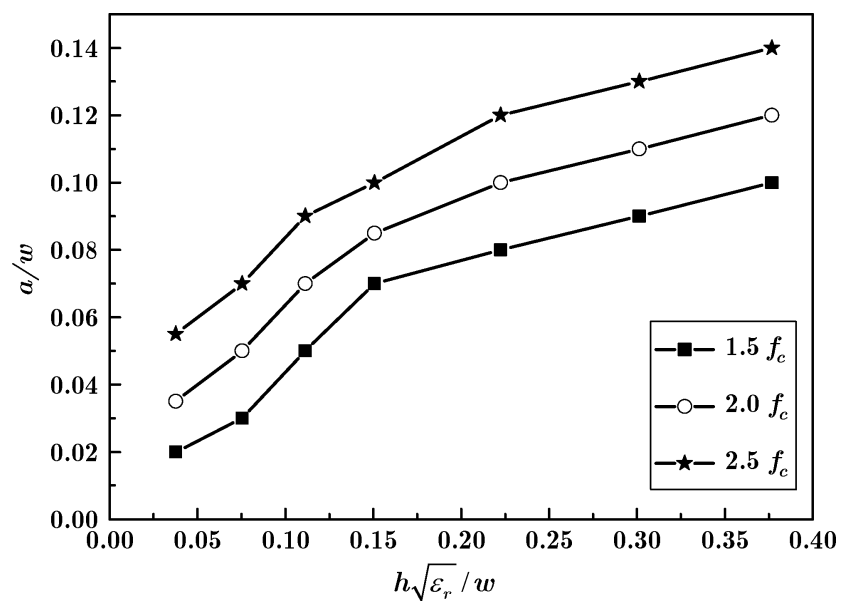

Fig. 4. Dependence of the normalized maximum electric field position $a / w$ on the electric height $h \sqrt{\varepsilon_{r}}$ normalized to the HMSIW width $w$. The curves correspond to results at different frequencies expressed in terms of cutoff frequency $f_{c}$.

in which $A_{p-0.5, q}$ is a constant, and $p$ and $q$ represent the numbers of extrema of the transverse electric field components in the $x$ - and $y$-direction, respectively. The parameters $k_{x,(p-0.5, q)}$, $k_{y,(p-0.5, q)}$, and $k_{z,(p-0.5, q)}$ are connected through the separation equation

$$
k_{x,(p-0.5, q)}^{2}+k_{y,(p-0.5, q)}^{2}+k_{z,(p-0.5, q)}^{2}=k_{0}^{2} \varepsilon_{r}
$$

where $k_{0}=(2 \pi f) / c$ is the free-space wavenumber with $f$ being the operation frequency and $c$ being the velocity of light in free space.

Considering that the HMSIW is mainly applied in singlemode operation, the following analysis is restricted to the fundamental quasi-TE $\mathrm{T}_{0.5,0}$ mode and the subscript $(0.5,0)$ is omitted for simplicity. The corresponding function $\psi$ is

$$
\psi \equiv \psi_{0.5,0}=A \cos k_{x}(w-x) e^{-j k_{z} z}, \quad 0 \leq x \leq w
$$

with

$$
\begin{aligned}
& k_{x}=\frac{\pi}{2(w-a)} \\
& k_{y}=0 \\
& k_{z}=\sqrt{k_{0}^{2} \varepsilon_{r}-\frac{\pi^{2}}{4(w-a)^{2}}}
\end{aligned}
$$

in which the parameter $a$ is the $x$-coordinate of the position of the maximum electric field along the cross section of the HMSIW. The dependence of the value $a / w$ on the frequency, on the substrate dielectric permittivity $\varepsilon_{r}$, and on the HMSIW width $w$ and height $h$ is demonstrated in Fig. 4. Data in this figure are obtained by simulating a number of HMSIW models of various $\varepsilon_{r} \in(2.2,15), h \in(0.254 \mathrm{~mm}, 2.54 \mathrm{~mm})$, and $w \in(2.5 \mathrm{~mm}, 10 \mathrm{~mm})$ with the help of the software CST Microwave Studio. It is noted that for fixed HMSIW dimensions, the position of the maximum electric field moves inwards the waveguide with increasing frequency.
Substituting (3) in the general equation [21, equ. (3-89)], the field components inside the HMSIW can be calculated as follows:

$$
\begin{aligned}
E_{y,(0.5,0)}=A k_{x} \sin k_{x}(w-x) e^{-j k_{z} z} \\
H_{x,(0.5,0)}=\frac{-A k_{x} k_{z}}{\omega \mu} \sin k_{x}(w-x) e^{-j k_{z} z} \\
H_{z,(0.5,0)=} \frac{A\left(\varepsilon_{r} k_{0}^{2}-k_{z}^{2}\right)}{j \omega \mu} \cos k_{x}(w-x) e^{-j k_{z} z}, \\
0 \leq x \leq w
\end{aligned}
$$

where $\omega$ is the phase frequency with the value of $\omega=2 \pi f$ and $\mu$ is the substrate permeability. The characteristic impedance of the dominant mode takes the form of the wave impedance and can be accordingly calculated as $Z_{c}=E_{y} / H_{x}=(-\omega \mu) / k_{z}$, as in the case of conventional rectangular waveguides [22].

The field in the homogeneous medium is mainly due to the radiation from the open aperture $(x=0)$. In principle, the equivalent electric and magnetic currents on the aperture can be analytically computed according to the field components given in (4). However, since the ratio of $a / w$ is close to 0 , which leads to $\cos k_{x} w \approx 0$, the equivalent electric current density $\vec{J}_{y}=2 \vec{a}_{x} \times\left.\vec{a}_{z} H_{z,(0.5,0)}\right|_{x=0}$ can be neglected. Therefore, only the equivalent magnetic current density $\vec{M}_{z}$ is considered in the following calculation of the field outside the HMSIW.

As depicted in Fig. 3, the points $P$ and $Q$ are the field observation point and source point, respectively. The equivalent magnetic current density $\vec{M}_{z}$ is calculated as [23]

$$
\begin{aligned}
\vec{M}_{z} & =-2 \vec{a}_{x} \times\left.\vec{a}_{y} E_{y I,(0.5,0)}\right|_{x=0} \\
& =-\vec{a}_{z} 2 A k_{x} \sin \left(k_{x} w\right) e^{-j k_{z} z} .
\end{aligned}
$$

where the factor 2 arises from the mirror effect of the ground plane.

Multiplying (5) with the Green function in the surrounding medium and integrating the product over the open aperture, i.e., $y^{\prime} \in(-h / 2, h / 2)$ and $z^{\prime} \in(-\infty,+\infty)$, the electric vector potential can be obtained as

$$
F_{z} \approx-\frac{A h k_{x}}{2 \pi} \sin \left(k_{x} w\right) e^{-j k_{z} z} \int_{-\Delta l}^{\Delta l} e^{-j k_{z} t} \frac{e^{-j k_{0, \mathrm{eff}} R}}{R} d t
$$

where $\Delta l \rightarrow \infty, t=z^{\prime}-z$, and $k_{0 \text {,eff }}=2 \pi f \sqrt{\varepsilon_{0, \mathrm{eff}}} / c$. In (6), $R$ represents the distance between the source point $Q$ and the field observation point $P$. The exact value of $R$ is $\sqrt{\rho^{2}+t_{1}^{2}}$, as shown in Fig. 3, but for simplicity, the approximation $R \approx$ $\sqrt{\rho^{2}+t^{2}}$ is adopted in the integration of (6) due to the small thickness $h$ of the substrate. Moreover, since the field intensity at point $P$ mainly depends on those source points in the near region, the integral can be limited to a finite value of $\Delta l$. In this study, it is empirically found that the minimum value of $\Delta l$ required for a satisfactory convergence of (6) is three to five times the wavelength in free space.

Based on (6), the field components can be calculated using $E_{\varphi}=\left(\partial F_{z} / \partial \rho\right)$ and $H_{z}=(j / \omega \mu)\left(\left(\partial^{2} F_{z} / \partial \rho^{2}\right)+\right.$ $\left.\left(\partial F_{z} / \rho \partial \rho\right)\right)$ in cylindrical coordinates [23].

The calculated electric field component $E_{y}$ on the xoz-plane is plotted in Fig. 2. The normalized electric field inside the 


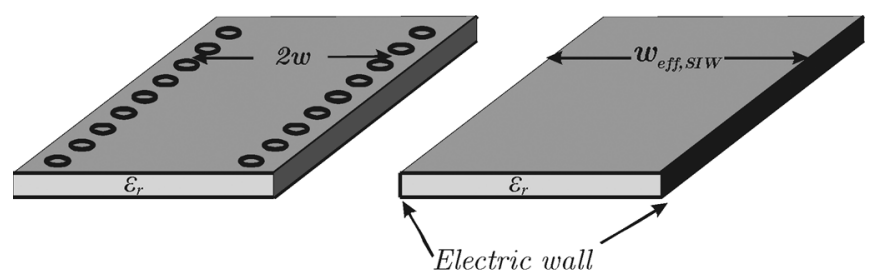

(a)

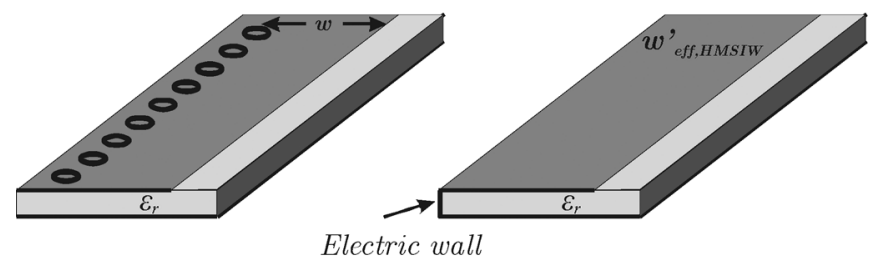

(b)

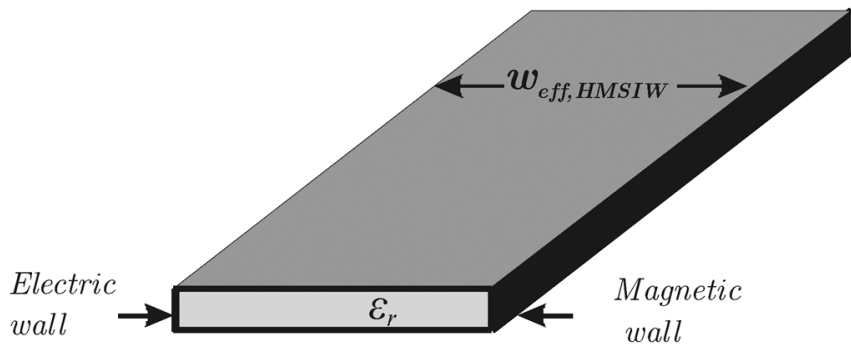

(c)

Fig. 5. (a) SIW with a width $2 w$ and the corresponding equivalent model (a conventional rectangular waveguide). (b) HMSIW as evolution from the SIW cut along its longitudinal symmetry plane, and the correspondingly cut equivalent model. (c) Second equivalent model of the HMSIW, proposed for the calculation of the phase constant.

HMSIW $(0<x / w<1)$ is given by dividing (4a) by the magnitude $\left|A k_{x}\right|$ and that outside the HMSIW $(x / w<0)$ is calculated through numerical integration of

$$
\begin{aligned}
\bar{E}_{\varphi}= & \frac{E_{\varphi}}{\left|A k_{x}\right|} \\
= & \frac{h \sin \left(k_{x} w\right)}{2 \pi} e^{-j k_{z} z} \\
& \times \int_{-\Delta l}^{\Delta l} \frac{\left(1+j k_{0, \mathrm{eff}} \sqrt{\rho^{2}+t^{2}}\right) \rho e^{-j k_{0, \mathrm{eff}} \sqrt{\rho^{2}+t^{2}}-j k_{z} t}}{\left(\rho^{2}+t^{2}\right)^{3 / 2}} d t .
\end{aligned}
$$

A good agreement between the calculation and simulation results is observed, which validates the field equations.

\section{B. Second Equivalent Model: Phase Constant}

As depicted in Fig. 5(a), a SIW with a width $2 w$ is equivalent, in terms of cutoff frequency, to a conventional rectangular waveguide with a width $w_{\text {eff,SIW }}$ when the widths $2 w$ and $w_{\text {eff,SIW }}$ satisfy the fitted equation [7, eq. (9)]

$$
w_{\text {eff,SIW }}=(2 w)-1.08 \frac{d^{2}}{s}+0.1 \frac{d^{2}}{(2 w)}
$$

where $d$ and $s$ are the vias' diameter and the spacing between adjacent vias, respectively. This equivalent rectangular waveguide has the same height $h$ and is filled with the same dielectric material as the SIW.
Cutting the SIW and its equivalent rectangular waveguide in half along their longitudinal symmetry planes, an HMSIW with a width $w$ and the corresponding equivalent waveguide with a width

$$
w_{\text {eff,HMSIW }}^{\prime}=w_{\text {eff,SIW } / 2}
$$

are obtained as shown in Fig. 5(b). It is noted that the equivalent model in Fig. 5(b) is an open structure. Therefore, due to the fringing fields, it is still difficult to calculate the cutoff frequency and phase constant of the HMSIW by directly using the width $w_{\text {eff,HMSIW }}^{\prime}$.

Considering, on one hand, that the electric field is mainly tangential to the open aperture where it approximately reaches its maximum (Fig. 2), and on the other hand, that the magnetic field is mainly perpendicular to the open aperture, a new equivalent waveguide is proposed with the open aperture replaced by a magnetic wall, as shown in Fig. 5(c). The width of this new equivalent model of the HMSIW is

$$
w_{\text {eff,HMSIW }}=w_{\text {eff,HMSIW }}^{\prime}+\Delta w
$$

where the additional width $\Delta w$, which still needs to be determined, accounts for the effect of the fringing fields and is a function of the width $w_{\text {eff,HMSIW }}^{\prime}$, of the waveguide height $h$, and of the substrate permittivity $\varepsilon_{r}$. Based on the present equivalent model, the cutoff frequency and phase constant of the quasi$\mathrm{TE}_{0.5,0}$ mode in the original HMSIW can be easily calculated as

$$
\begin{aligned}
f_{c, \mathrm{TE}_{0.5,0}} & =\frac{c}{4 \sqrt{\varepsilon_{r}} w_{\mathrm{eff}, \mathrm{HMSIW}}} \\
k_{z, \mathrm{TE}_{0.5,0}} & =\sqrt{k_{0}^{2} \varepsilon_{r}-\left(\frac{\pi}{2 w_{\mathrm{eff}, \mathrm{HMSIW}}}\right)^{2}}
\end{aligned}
$$

with $k_{0}$ defined as in (2).

To determine the additional width $\Delta w$, the following procedure is carried out. Firstly, two HMSIWs [such as in Fig. 1(a)] with an identical profile, but different lengths, are modeled and simulated in HFSS. The resulting scattering parameters are processed using the multiline method (ML) [24], [25] in

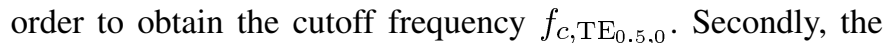
obtained $f_{c, \mathrm{TE}_{0.5,0}}$ is substituted into (11) to calculate the width $w_{\text {eff,HMSIW }}$ of the equivalent waveguide. Thirdly, the equivalent width $w_{\text {eff,HMSIw }}^{\prime}$ is calculated using (8) and (9), according to the metal posts configuration of the HMSIW. Finally, the additional width $\Delta w$ can be computed by substituting the obtained equivalent widths $w_{\text {eff,HMSIW }}$ and $w_{\text {eff,HMSIW }}^{\prime}$ into (10).

Varying the values of the HMSIW width $w$, of the HMSIW height $h$, and of the substrate permittivity $\varepsilon_{r}$ and iterating the procedure above, different sets of curves can be obtained describing $\Delta w$ versus $w_{\text {eff,HMSIW }}^{\prime}, \Delta w$ versus $h$, or $\Delta w$ versus $\varepsilon_{r}$. Fitting all curves with the nonlinear least squares method [26], the additional width $\Delta w$ normalized to the HMSIW height $h$ can be estimated as

$$
\begin{aligned}
\frac{\Delta w}{h}= & \left(0.05+\frac{0.30}{\varepsilon_{r}}\right) \\
\times \ln ( & 0.79 \frac{w_{\text {eff,HMSIW }}^{\prime 2}}{h^{3}}+\frac{104 w_{\text {eff,HMSIW }}^{\prime}-261}{h^{2}} \\
& \left.+\frac{38}{h}+2.77\right) .
\end{aligned}
$$




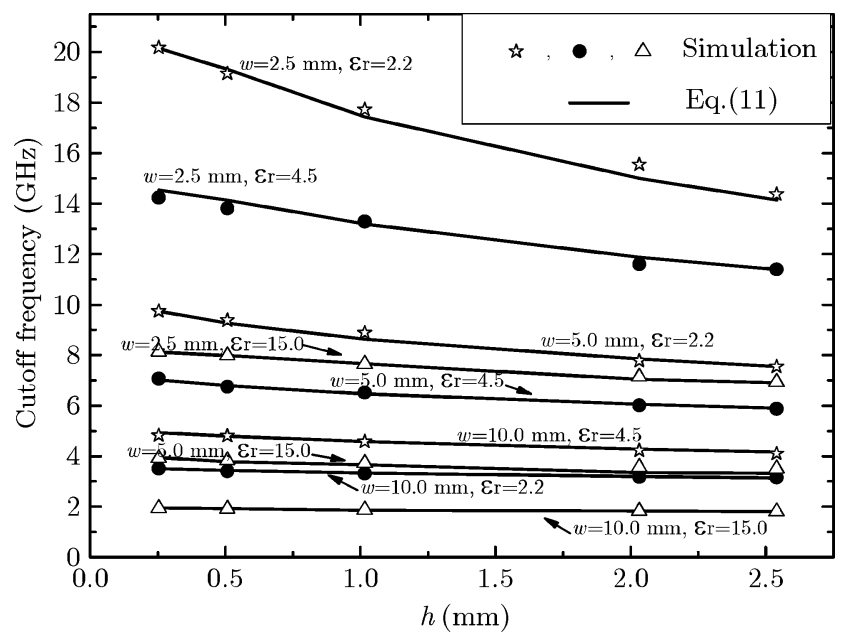

Fig. 6. Cutoff frequency versus the HMSIW height for variations of the HMSIW width and substrate permittivity. In all cases, the diameter $d$ and the spacing $s$ of the vias are 0.5 and $0.6 \mathrm{~mm}$, respectively.

This expression is valid for $\varepsilon_{r} \in(2.2,15)$, $h \in(0.254 \mathrm{~mm}, 2.54 \mathrm{~mm})$, and $w \in(2.5 \mathrm{~mm}, 10 \mathrm{~mm})$ covering a potential range of operation frequency from 2 to $60 \mathrm{GHz}$.

At this point, all necessary design formulas are available. For the practical synthesis of an HMSIW with a specified cutoff frequency, where the thickness $h$ and the substrate permittivity $\varepsilon_{r}$ of the printed circuit board are given, the equivalent width $w_{\text {eff,HMSIW }}$ is first determined using (11). Subsequently, the proper diameter $d$ and the spacing $s$ of the metallic posts are selected according to the operation frequency range [7]. Finally, substituting the known parameters $h, \varepsilon_{r}, w_{\text {eff.HMSIW }}, d$, and $s$ into (8)-(10) and (13), the HMSIW physical width $w$ (as the only unknown) can be obtained by solving those four equations simultaneously.

As shown in Fig. 6, the cutoff frequencies calculated through this procedure for various HMSIW widths, heights, and substrate permittivities have been compared to those obtained from simulations of the corresponding devices. For all the examined examples in Fig. 6, the diameter and spacing of the vias in the HMSIW are $d=0.5 \mathrm{~mm}$ and $s=0.6 \mathrm{~mm}$, respectively. In the mentioned valid range of the design formulas, the calculation error is estimated below $2 \%$.

Furthermore, for the two HMSIW prototypes mentioned at the beginning of this section, the phase constants have been calculated using (12) and compared with the simulation and measurement results, as plotted in Figs. 7 and 8. The agreement between all results in Figs. 6-8 validates the proposed design formulas (8)-(10) and (13).

Using the present equivalent model, the frequency range for the single mode operation can also be estimated based on the calculation of the cutoff frequencies for the fundamental quasi- $\mathrm{TE}_{0.5,0}$ mode [as given in (11)] and the first higher order quasi- $\mathrm{TE}_{1.5,0}$ mode. In the closed equivalent waveguide, the cutoff frequency of the quasi- $\mathrm{TE}_{1.5,0}$ mode can be approximated as

$$
f_{c, \mathrm{TE}_{1.5,0}}=\frac{3 c}{4 \sqrt{\varepsilon_{r}} w_{\mathrm{eff}, \mathrm{HMSIW}}}
$$

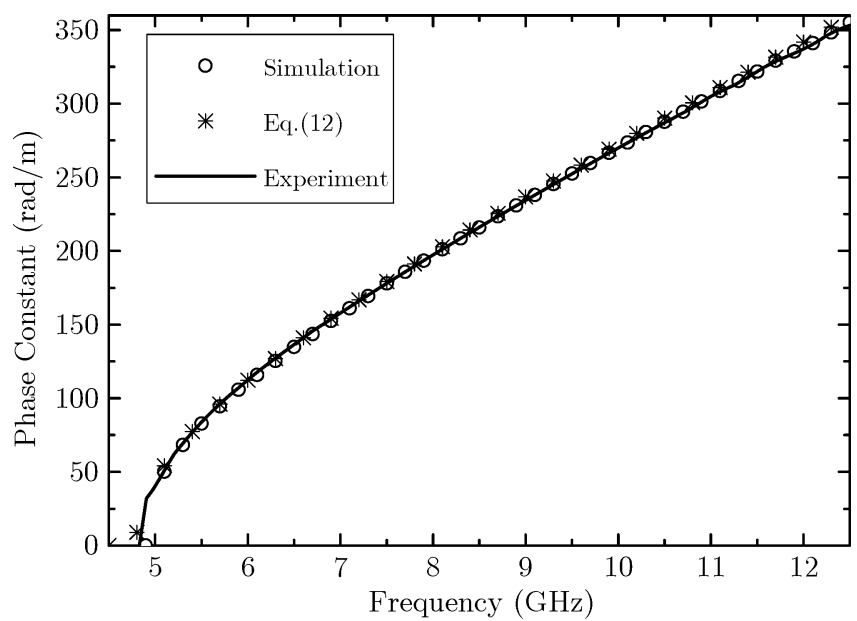

Fig. 7. Phase constant of the quasi-TE $E_{0.5,0}$ mode in the HMSIW with a width $w=10.0 \mathrm{~mm}$, height $h=0.508 \mathrm{~mm}$, and substrate permittivity $\varepsilon_{r}=2.2$. The diameter $d$ and the spacing $s$ of the vias are 0.5 and $0.6 \mathrm{~mm}$, respectively.

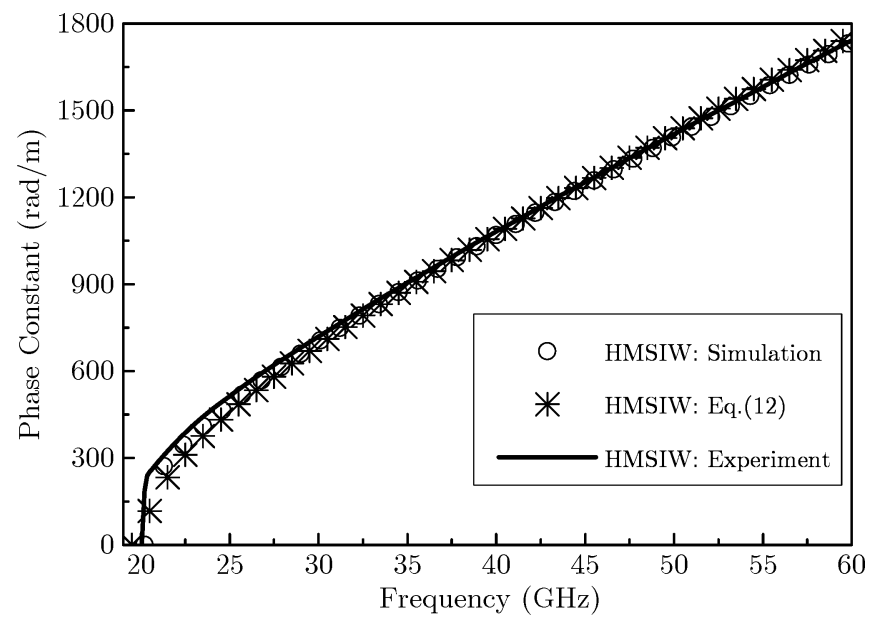

Fig. 8. Phase constant of the quasi-TE $\mathrm{T}_{0.5,0}$ mode in the HMSIW with a width $w=2.5 \mathrm{~mm}$, height $h=0.254 \mathrm{~mm}$, and substrate permittivity $\varepsilon_{r}=2.2$. The diameter $d$ and the spacing $s$ of the vias are 0.5 and $0.6 \mathrm{~mm}$, respectively.

which is three times that of the fundamental mode. Therefore, if not considering any suppression of higher modes through feeding technologies, an HMSIW with a width $w$ yields a singlemode operation frequency range approximately twice that of a SIW with a width $2 w$.

\section{ATtENUATION}

In this section, the attenuation of the HMSIW is investigated numerically and experimentally. First, a comparison of the simulated results with the experimental data is provided as an assessment of the simulation accuracy. A procedure is then presented for numerically analyzing the loss mechanism of the HMSIW in the frequency range of $20-60 \mathrm{GHz}$. Finally, the measured losses of the HMSIW are compared to those of the SIW and the standard MS line in a representative case.

All HMSIW models used in the simulations and all the fabricated HMSIW devices are corresponding to the second prototype mentioned at the beginning of Section II and operate in the frequency range of 20-60 GHz. In those models and devices, the feeding MS lines and transitions are kept identical, but the 


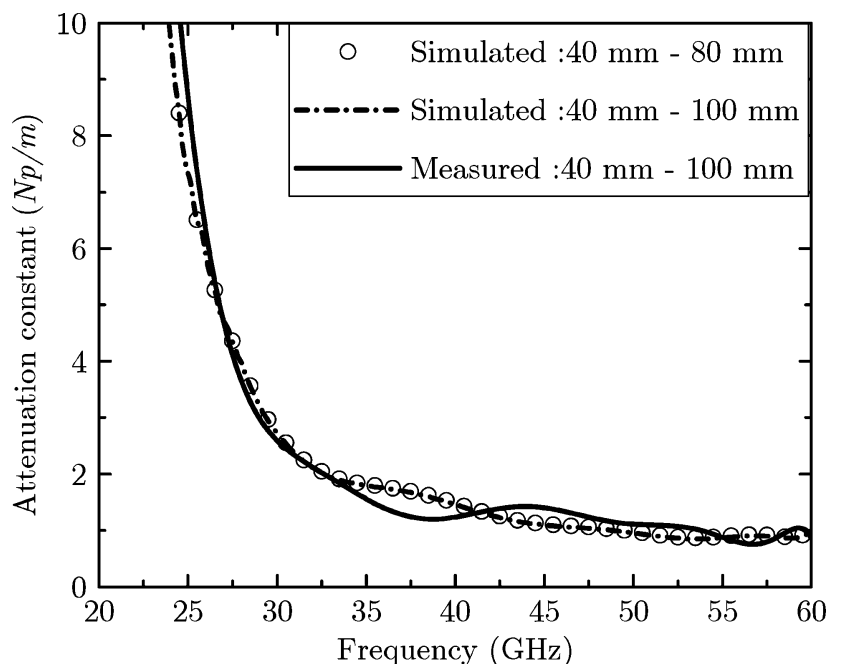

Fig. 9. Simulated and measured attenuation constant of the HMSIW with a width $w=2.5 \mathrm{~mm}$, height $h=0.254 \mathrm{~mm}$, and substrate permittivity $\varepsilon_{r}=$ 2.2. The diameter $d$ and the spacing $s$ of the vias are 0.5 and $0.6 \mathrm{~mm}$, respectively. All curves are obtained by applying the ML method to lines of different lengths, as given in the legend.

middle HMSIW length [marked as $l$ in Fig. 1(a)] is varied for the ML analysis.

\section{A. Validation of the Simulation}

Since the analysis of the loss mechanism in the HMSIW is based on simulations, the accuracy of the simulation tool has been firstly assessed. For this purpose, three HMSIW prototypes with lengths $l=40 \mathrm{~mm}, l=80 \mathrm{~mm}$, and $l=100 \mathrm{~mm}$ were first analyzed using HFSS. The attenuation constant was then calculated by comparing the losses of the HMSIWs with lengths $l=40 \mathrm{~mm}$, and $l=80 \mathrm{~mm}$ using the ML method. Similarly, the attenuation constant can also be computed by comparing the losses of the HMSIWs with lengths $l=40 \mathrm{~mm}$ and $l=100 \mathrm{~mm}$. The attenuation constant obtained through both computations is presented in Fig. 9. It can be seen that the results of both cases are consistent, indicating that the simulations and calculations are convergent. A quantitative analysis shows that the difference between the two curves is within $0.04 \mathrm{~Np} / \mathrm{m}$.

On the experimental side, HMSIW prototypes with lengths $l=40 \mathrm{~mm}$ and $l=100 \mathrm{~mm}$ were fabricated and measured. The experimentally obtained attenuation constant is also plotted in Fig. 9. A fairly good agreement is achieved between the simulated and measured results over the entire band of interest. This further confirms the accuracy of the simulation method and provides justification for the following numerical analysis.

\section{B. Numerical Investigation of the Losses in HMSIW}

To characterize the loss mechanism in the HMSIW, the same two HMSIWs with $l=40 \mathrm{~mm}$ and $l=100 \mathrm{~mm}$ are simulated again, yet with altered properties of the materials, i.e., the conductivity of the metal and the loss tangent of the dielectric are varied. This procedure allows distinguishing between the conductive, dielectric, and radiation losses in the HMSIW and provides a qualitative characterization of their variation with frequency.

1) Radiation Losses: To determine the total power leakage from both the open aperture and the space between the vias, all

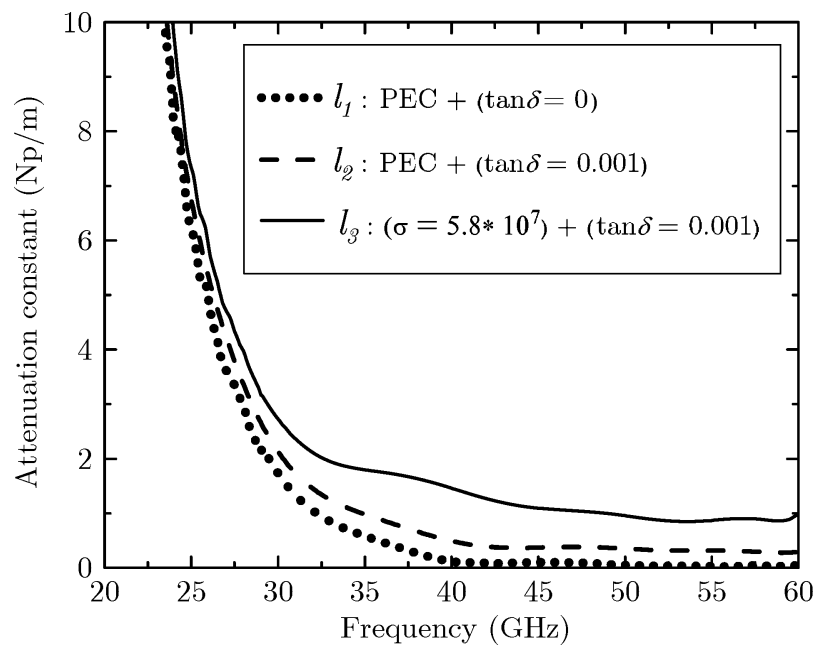

Fig. 10. Simulated attenuation constant of the quasi-TE $E_{0.5,0}$ mode in the HMSIW with varied conductivity of the conductor and loss tangent of the dielectric substrate. The dimensions of the HMSIW are $w=2.5 \mathrm{~mm}$, $h=0.254 \mathrm{~mm}, d=0.5 \mathrm{~mm}$, and $s=0.6 \mathrm{~mm}$. The substrate permittivity is $\varepsilon_{r}=2.2$. The label PEC denotes perfect electric conductor $(\sigma=\infty)$.

the materials used in the HMSIW simulation models are first set to be lossless, i.e., the conductivity of the metal is set to $\sigma=\infty$ and the loss tangent of the dielectric to $\tan \delta=0$. The obtained attenuation constant is shown as the curve $l_{1}$ in Fig. 10. Since there are no conductive or dielectric losses in the model, the attenuation constant completely characterizes the power leakage from the HMSIW. It can be observed that those radiation losses become extremely high as the frequency is decreased toward the cutoff. The reason for this performance degradation can be intuitively understood when considering that at the cutoff frequency, the open side of the HMSIW can be viewed as a slot with constant field distribution.

However, as frequency exceeds $40 \mathrm{GHz}$, the attenuation constant exclusively resulting from the radiation losses drops to below $0.1 \mathrm{~Np} / \mathrm{m}$, indicating a small contribution to the total attenuation. This corroborates the observation in Fig. 8 that the nonradiative equivalent model, as given in Fig. 5(c) and (12), can approximate the HMSIW more accurately at higher frequencies than close to cutoff.

2) Dielectric Losses: Maintaining the metallic material to be lossless, realistic dielectric losses are introduced in the HMSIW simulation models as loss tangent set to $\tan \delta=0.001$. The resulting simulated attenuation constant as a function of the frequency is plotted as $l_{2}$ in Fig. 10. The increased attenuation compared to the lossless case is obviously caused by dielectric losses in the HMSIW, which, therefore, can be directly characterized as the difference between the curves $l_{1}$ and $l_{2}$.

From Fig. 10, it can be found that the effect of the dielectric losses is masked by radiation losses at frequencies below $25 \mathrm{GHz}$. As frequency increases, the two curves $l_{1}$ and $l_{2}$ get visibly separated from each other and reveal the effect of dielectric losses. Above $35 \mathrm{GHz}$, the dielectric losses change only slightly with the frequency for the currently used substrate material of stable dispersion properties. More specifically, the difference between curves $l_{1}$ and $l_{2}$ remains at a level of $0.3 \pm$ $0.1 \mathrm{~Np} / \mathrm{m}$ above $35 \mathrm{GHz}$. 


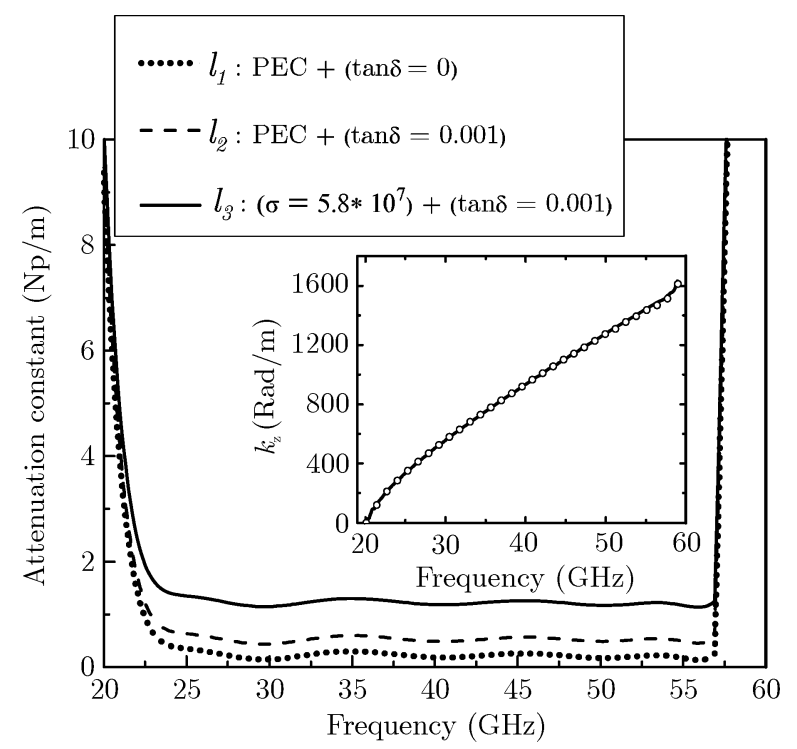

Fig. 11. Simulated attenuation constant of the quasi-TE $E_{10}$ mode in the SIW with varied conductivity of the conductor and loss tangent of the dielectric substrate. The SIW width and height are 5.45 and $0.254 \mathrm{~mm}$. The diameter and spacing of the vias are 0.5 and $0.6 \mathrm{~mm}$, respectively. The substrate permittivity is $\varepsilon_{r}=2.2$. The label PEC denotes perfect electric conductor $(\sigma=\infty)$. The inset shows the corresponding phase constant obtained from simulations (solid line) and measurements (discrete circles).

3) Conductive Losses: Finally, both the conductor and dielectric used in the simulated models of the HMSIW are set to be the realistic lossy materials, i.e., with the conductivity $\sigma=5.8 \times 10^{7} \mathrm{~S} \cdot \mathrm{m}^{-1}$ for the metal and the loss tangent $\tan \delta=0.001$ for the dielectric substrate. The attenuation constant for this case is plotted as the curve $l_{3}$ in Fig. 10, and reflects the combined effects of the conductive, dielectric, and radiation losses. The difference between the curves $l_{2}$ and $l_{3}$ arises from the added conductive losses in this prototype. Therefore, comparison of the curve $l_{3}$ to $l_{2}$ leads to the conclusion that conductive losses can be almost negligible at frequencies lower than $28 \mathrm{GHz}$ when compared to radiation losses. However, with the further increase of frequency, the conductive losses become the dominant cause of attenuation in the HMSIW if a low-dispersion low-loss substrate material is used.

4) Comparison to Losses in the SIW: For comparison, two SIWs with lengths of $l=40 \mathrm{~mm}$ and $l=100 \mathrm{~mm}$ fed by a centered MS line have been designed using the same substrate as the second HMSIW prototype (20-60 GHz). The radius and spacing of the vias in the SIWs are the same as those in the HMSIW. The width of the SIWs is determined to be $2 w=5.45 \mathrm{~mm}$ in order to obtain the same cutoff frequency as the HMSIW, as indicated in the inset of Fig. 11. Furthermore, the symmetry granted by the centered feeding line suppresses all the even modes, thereby providing the SIW with a single-mode operation frequency range approximately matching that of the HMSIW.

The losses in the SIW are analyzed numerically by applying the same procedure as for the HMSIW. The obtained attenuation constants corresponding to the fundamental quasi- $\mathrm{TE}_{10}$ mode are shown in Fig. 11. The drastic increase in the attenuation at frequencies above $58 \mathrm{GHz}$ results from the occurrence of the bandstop [7]. For the present SIW design, the findings are similar to those for the HMSIW and can be summarized

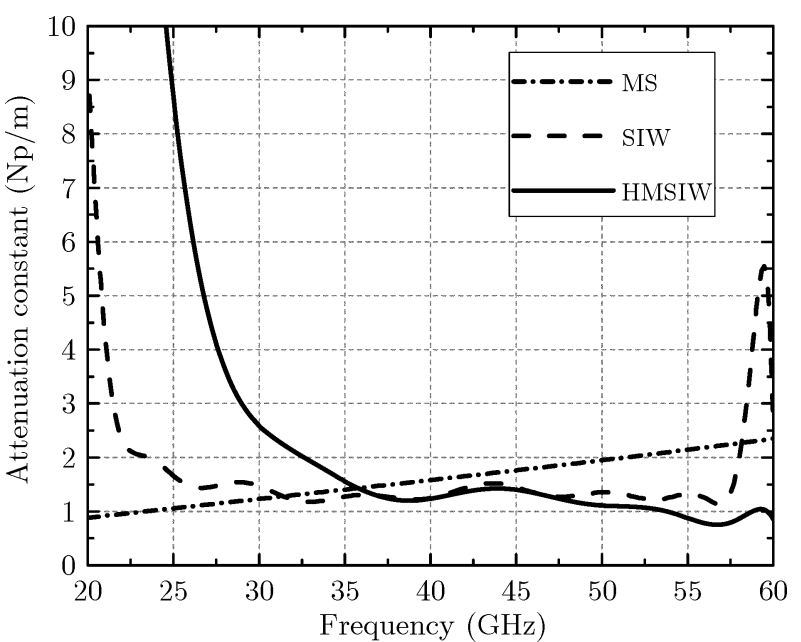

Fig. 12. Measured attenuation constants of the MS line, SIW, and HMSIW.

as follows. The dominant attenuation is caused by the conductive losses. The dielectric losses are quite low for the presently used low-loss dielectric substrate. The radiation losses are almost negligible when the waveguide operates far above cutoff. The increase of radiation losses near cutoff is less pronounced than in the HMSIW, as there is no fully open side in the SIW.

\section{Experimental Comparison of HMSIW With SIW and Microstrip}

For a direct experimental comparison with the second HMSIW prototype, the SIWs studied above and two standard 50- $\Omega$ MS lines of lengths of 50.8 and $299.3 \mathrm{~mm}$ have also been fabricated. The MS lines were manufactured on the same substrate as the SIWs and HMSIWs. A time gating technique was used in the measurements in order to exclude the effects of the connectors. The measured attenuation constants are given in Fig. 12.

The attenuation of the MS line increases monotonically with the frequency and is lower than that of the SIW and HMSIW at frequencies below $35 \mathrm{GHz}$. This is explained by the large radiation losses occurring around the cutoff frequency of the SIW and HMSIW. As the frequency increases, both the conductive losses and radiation losses increase in the MS line. As a consequence, the attenuation of the MS line exceeds those of the SIW and HMSIW at frequencies above $35 \mathrm{GHz}$.

Comparing the losses of the SIW and HMSIW indicates that the attenuation of the HMSIW is higher than that of the SIW at frequencies close to the cutoff, but becomes lower as the frequency increases above $40 \mathrm{GHz}$. This can be explained by considering the results in Figs. 10 and 11.

1) At frequencies below $40 \mathrm{GHz}$, the conductive and dielectric losses of the SIW are comparable to those of the HMSIW. However, with the same cutoff frequency, the radiation losses of the HMSIW are much higher than those of the SIW due to the power leakage from the open side of the HMSIW.

2) At frequencies above $40 \mathrm{GHz}$, the radiation losses of the HMSIW are reduced to a level comparable to those of the SIW, and so are the dielectric losses. The conductive losses become dominant for both lines in this frequency range. In the absence of fringing at the open side, the conductive 
losses in the HMSIW would be equal to those in the SIW. However, as the frequency increases, more energy propagates along the waveguide in the fringing region, where the fields are not affected by the conductors. Consequently, a lower attenuation is achieved in the HMSIW.

Although the comparison of the attenuation above is based on the study of specific examples rather than general models, this work with no loss of generality presents a method to analyze the attenuation of a transmission line through the separation of the conductive, dielectric, and radiation losses from the total attenuation. The presented measurement results demonstrate a practically achievable level of attenuation in the HMSIW for the frequency range of $20-60 \mathrm{GHz}$.

Before concluding, a general remark on substrate selection in the perspective of this study is required. In a typical practical design procedure, an appropriate substrate is generally determined in advance according to certain specifications such as the operation frequency band. Subsequently, the type of transmission line is selected, by considering the losses, volume, fabrication cost, etc. Therefore, the use of an identical substrate has been assumed here as a preliminary condition for a fair comparison of the losses between the MS line, SIW, and HMSIW.

However, in a case where there are no $a$ priori restrictions on the choice of substrate, other considerations need to be taken into account. In particular, increasing the height of the SIW can significantly reduce the conductive losses in comparison with the MS line [27]. This strategy is not viable for the HMSIW since the height is strictly constrained by the operation frequency band and cannot be enlarged arbitrarily. Additionally, a general drawback of the HMSIW and the SIW is the larger volume compared with the MS line. Therefore, due to their specific advantages and drawbacks, either the MS line, SIW, or HMSIW may be preferred in different applications.

\section{CONCLUSION}

The propagation properties of the HMSIW, i.e., the phase constant and attenuation constant, have been investigated using theoretical, numerical, and experimental methods. Two equivalent models have been proposed to analyze the field distribution and calculate the phase constant of the HMSIW. An approximate design equation has been derived using a statistical method and has been validated by numerical and experimental results. Additionally, the attenuation characteristics of the HMSIW have been studied through a numerical separation of the conductive, dielectric, and radiation losses. It is found that for identical materials and arrangement of the vias, the losses in the HMSIW can be at the same level or even lower compared to those of the SIW. Further, in the millimeter-wave range, both the HMSIW and SIW exhibit lower attenuation compared to a standard MS line made of the same materials. To make the comparison as reasonable as possible, the selected dimensions and materials in all investigated examples of transmission lines are quite common in the sense of practical applications.

\section{ACKNOWLEDGMENT}

The authors are grateful for the support of their colleagues in the Electromagnetic Fields and Microwave Electronics Laboratory, ETH Zürich, Zürich, Switzerland. In particular, the authors thank H. Benedickter and G. Almpanis, for their helpful discussions with the design and measurement. The authors also appreciate M. Lanz, and C. Maccio, for their perfect manufacture of the devices.

\section{REFERENCES}

[1] D. Deslandes and K. Wu, "Integrated microstrip and rectangular waveguide in planar form," IEEE Microw. Wireless Compon. Lett., vol. 11, no. 2 , pp. $68-70$, Feb. 2001.

[2] J. Hirokawa and M. Ando, "Single-layer feed waveguide consisting of posts for plane TEM wave excitation in parallel plates," IEEE Trans. Antennas Propag., vol. 46, no. 5, pp. 625-630, May 1998.

[3] A. Piloto, K. Leahy, B. Flanick, and K. A. Zaki, "Waveguide filters having a layered dielectric structures," U.S. Patent 5382 931, Jan. 17, 1995.

[4] Y. Cassivi and K. Wu, "Dispersion characteristics of substrate integrated rectangular waveguide," IEEE Microw. Wireless Compon. Lett., vol. 12 , no. 9, pp. 333-335, Sep. 2002.

[5] F. Xu, Y. L. Zhang, W. Hong, K. Wu, and T. J. Cui, "Finite-difference frequency-domain algorithm for modeling guided-wave properties of substrate integrated waveguide," IEEE Trans. Microw. Theory Tech., vol. 51, no. 11, pp. 2221-2227, Nov. 2003.

[6] L. Yan, W. Hong, K. Wu, and T. J. Cui, "Investigations on the propagation characteristics of the substrate integrated waveguide based on the method of lines," Proc. Inst. Elect. Eng.-Microw., Antennas, Propag., vol. 152, no. 1, pt. H, pp. 35-42, Feb. 2005.

[7] F. Xu and K. Wu, "Guided-wave and leakage characteristics of substrate integrated waveguide," IEEE Trans. Microw. Theory Tech., vol. 53, no. 1, pp. 66-72, Jan. 2005.

[8] L. Yan, W. Hong, G. Hua, J. X. Chen, K. Wu, and T. J. Cui, "Simulation and experiment on SIW slot array antennas," IEEE Microw. Wireless Compon. Lett., vol. 14, pp. 446-448, Sep. 2004.

[9] Y. L. Zhang, W. Hong, K. Wu, J. X. Chen, and H. J. Tang, "Novel substrate integrated waveguide cavity filter with defected ground structure," IEEE Trans. Microw. Theory Tech., vol. 53, no. 4, pp. 1280-1287, Apr. 2005.

[10] Z. C. Hao, W. Hong, J. X. Chen, X. P. Chen, and K. Wu, "Compact super-wide bandpass substrate integrated waveguide (SIW) filters," IEEE Trans. Microw. Theory Tech., vol. 53, no. 9, pp. 2968-2977, Sep. 2005.

[11] H. J. Tang, W. Hong, J.-X. Chen, G. Q. Luo, and K. Wu, "Development of millimeter-wave planar diplexers based on complementary characters of dual-mode substrate integrated waveguide filters with circular and elliptic cavities," IEEE Trans. Microw. Theory Tech., vol. 55, no. 4, pp. 776-782, Apr. 2007.

[12] E. Moldovan, R. G. Bosisio, and K. Wu, " $W$-band multiport substrateintegrated waveguide circuits," IEEE Trans. Microw. Theory Tech., vol. 54, no. 2, pp. 625-632, Feb. 2006.

[13] N. Grigoropoulos and P. R. Young, "Compact folded waveguides," in 34th Eur. Microw. Conf., Amsterdam, The Netherlands, 2004, pp. 973-976.

[14] N. Grigoropoulos, B. Sanz-Izquierdo, and P. R. Young, "Substrate integrated folded waveguides (SIFW) and filters," IEEE Microw. Wireless Compon. Lett., vol. 15, no. 12, pp. 829-831, Dec. 2005.

[15] W. Che, L. Geng, K. Deng, and Y. L. Chow, "Analysis and experiments of compact folded substrate integrated waveguide," IEEE Trans. Microw. Theory Tech, vol. 56, no. 1, pp. 88-93, Jan. 2008.

[16] W. Hong, B. Liu, Y. Q. Wang, Q. H. Lai, and K. Wu, "Half mode substrate integrated waveguide: A new guided wave structure for microwave and millimeter wave application," in Proc. Joint 31 st Int. Infrared Millimeter Wave Conf./14th Int. Terahertz Electron. Conf., Shanghai, China, Sep. 2006, pp. 18-22.

[17] B. Liu, W. Hong, Y. Q. Wang, Q. H. Lai, and K. Wu, "Half mode substrate integrated waveguide (HMSIW) $3 \mathrm{~dB}$ coupler," IEEE Microw. Wireless Compon. Lett., vol. 17, no. 1, pp. 22-24, Jan. 2007.

[18] Y. Wang, W. Hong, Y. D. Dong, and B. Liu et al., "Half mode substrate integrated waveguide (HMSIW) bandpass filter," IEEE Microw. Wireless Compon. Lett., vol. 17, no. 4, pp. 256-267, Apr. 2007.

[19] B. Liu, W. Hong, Y. Zhang, H. J. Tang, X. X. Yin, and K. Wu, "Half mode substrate integrated waveguide 180 3-dB directional couplers," IEEE Trans. Microw. Theory Tech., vol. 55, no. 12, pp. 2586-2592, Dec. 2007. 
[20] Y. Cheng, W. Hong, and K. Wu, "Half mode substrate integrated waveguide (HMSIW) directional filter," IEEE Microw. Wireless Compon. Lett., vol. 17, no. 7, pp. 504-506, Jul. 2007.

[21] R. F. Harrington, Time-Harmonic Electromagnetic Field. New York: McGraw-Hill, 1961, pp. 129-130.

[22] R. F. Harrington, Time-Harmonic Electromagnetic Field. New York: McGraw-Hill, 1961, pp. 66-74.

[23] R. F. Harrington, Time-Harmonic Electromagnetic Field. New York: McGraw-Hill, 1961, pp. 98-106.

[24] R. B. Marks, "A multiline method of network analyzer calibration," IEEE Trans. Microw. Theory Tech., vol. 39, no. 7, pp. 1205-1215, Jul. 1991.

[25] G. F. Engen and C. A. Hoer, "Thru-reflect-line: An improved technique for calibrating the dual six-port automatic network analyzer," IEEE Trans. Microw. Theory Tech., vol. MTT-37, no. 12, pp. 987-993, Dec. 1979.

[26] T. Sauer, Numerical Analysis. Upper Saddle River, NJ: Pearson Educ., 2006, pp. 230-235.

[27] M. Bozzi, F. Xu, L. Perregrimi, and K. Wu, "Circuit modeling and physical interpretation of substrate integrated waveguide structures of millimeter wave applications," Int. J. Microw. Opt. Tech., vol. 3, no. 3, pp. 329-338, Jul. 2008.

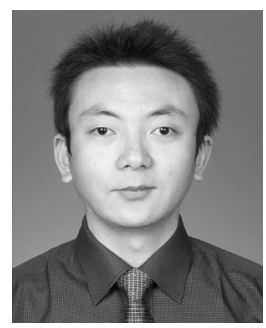

Qinghua Lai (S'09) received the B.S. degree in radio engineering from the University of Electronic Science and Technology, Chengdu, China, in 2004, and is currently working toward the M. Phi. and Ph.D. degrees in radio engineering at Southeast University, Nanjing, China.

From July 2007 to June 2008, he was Visiting Student with ETH Zürich, Zürich, Switzerland, where he was engaged in research on the radiation efficiency of dielectric resonator antennas and on the propagation properties of HMSIWs. Since September 2008, he has been an Academic Guest with ETH Zürich, where he is involved with the development of high-performance filters for base stations. His current research interests focus on array antennas and filters.

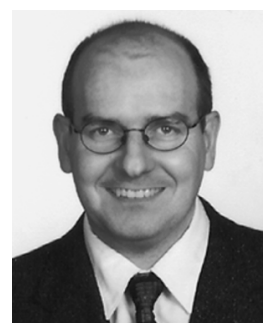

Christophe Fumeaux (M'03-SM'09) received the Diploma and Ph.D. degrees in physics from the ETH Zürich, Zürich, Switzerland, in 1992 and 1997, respectively.

From 1998 to 2000, he was a Post-Doctoral Researcher with the School of Optics, University of Central Florida, Orlando. In 2000, he joined the Swiss Federal Office of Metrology, Bern, Switzerland, as a Scientific Staff Member. From 2001 to 2008, he was a Research Associate and Group Leader with the Laboratory for Electromagnetic Fields and Microwave Electronics (IFH), ETH, Zürich. During Fall 2005, he was a Visiting Scientist with the LASMEA, University Blaise Pascal, Clermont-Ferrand, France. In 2008, he joined the School of Electrical and Electronic Engineering, University of Adelaide, Adelaide, South Australia, where he is currently an Associate Professor. His current main research interests concern computational electromagnetics, microwave circuits, and antenna engineering.

Dr. Fumeaux was the recipient of the ETH Silver Medal of Excellence for his doctoral dissertation. From 2006 to 2008, he was the chairman of the IEEE Swiss Joint Chapter on Microwave Theory and Techniques, Antennas and Propagation, and Electromagnetic Compatibility (EMC). He was the corecipient of the 2004 Outstanding Paper Award of the Applied Computational Electromagnetics Society (ACES).

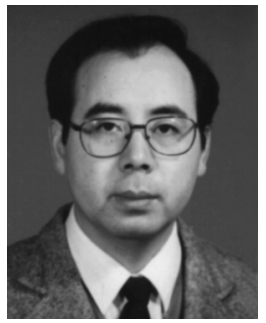

Wei Hong (M'92-SM'07) received the B.S. degree from the University of Information Engineering, Zhengzhou, China, in 1982, and the M.S. and Ph.D. degrees from Southeast University, Nanjing, China, in 1985 and 1988, respectively, all in radio engineering.

Since 1988, he has been with the State Key Laboratory of Millimeter Waves, Southeast University, where he is currently a Professor with the School of Information Science and Engineering. In 1993 and 1995-1998, he was a short-term Visiting Scholar with the University of California at Berkeley and the University of California at Santa Cruz. He has been engaged in numerical methods for electromagnetic problems, millimeter-wave theory and technology, antennas, electromagnetic scattering, RF technology for mobile communications, etc. He has authored or coauthored over 200 technical publications. He authored Principle and Application of the Method of Lines (in Chinese) (Southeast Univ. Press, 1993) and Domain Decomposition Methods for Electromagnetic Problems (in Chinese) (Sci. Press, 2005). He has served as a reviewer for IET Microwave, Antennas, and Propagation and Electronics Letters.

Dr. Hong is a Senior Member of the Chinese Institute of Electronics (CIE). $\mathrm{He}$ is vice president of the Microwave Society and Antenna Society, CIE. He has served as a reviewer for the IEEE TRANSACTIONS ON MICROWAVE THEORY AND TECHNiQues and the IEEE TRANSACTIONS ON ANTENNAS AND PROPAGATION. $\mathrm{He}$ is an associate editor for the IEEE TRANSACTIONS ON MICROWAVE THEORY AND TECHNIQUE. He was a two-time recipient of the 1992 and 1994 First-Class Science and Technology Progress Prizes presented by the Ministry of Education of China and the 1991 Fourth-Class National Natural Science Prize. He was also the recipient of the Foundations for China Distinguished Young Investigators and for the "Innovation Group" presented by the National Natural Science Foundation of China (NSFC).

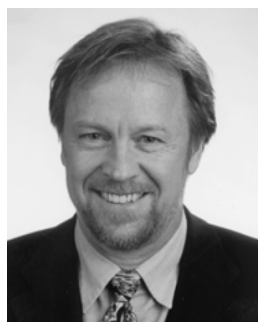

Rüdiger Vahldieck (M'85-SM'86-F'99) received the Dipl.-Ing. and Dr.-Ing. degrees in electrical engineering from the University of Bremen, Bremen, Germany, in 1980 and 1983, respectively.

He was a Postdoctoral Fellow with the University of Ottawa, Ottawa, ON, Canada, until 1986. In 1986, he joined the Department of Electrical and Computer Engineering, University of Victoria, Victoria, BC, Canada, where he became a Full Professor in 1991. During Fall and Spring 1992-1993, he was a Visiting Scientist with the Ferdinand-Braun-Insitute for Hochfrequenztechnik, Berlin, Berlin, Germany. In 1997, he became a Professor of electromagnetic field theory with the Swiss Federal Institute of Technology, Zürich, Switzerland, and became Head of the Laboratory for Electromagnetic Fields and Microwave Electronics (IFH) in 2003. In 2005, he became President of the Research Foundation for Mobile Communications and was elected Head of the Department of Information Technology and Electrical Engineering (D-ITET), ETH Zürich. Since 1981 he has authored or coauthored over 300 technical papers in books, journals, and conferences. His research interests include computational electromagnetics in the general area of electromagnetic compatibility (EMC), in particular, for computer-aided design of microwave, millimeter-wave, and opto-electronic integrated circuits.

Dr. Vahldieck is the past president of the 2000 IEEE International Zürich Seminar on Broadband Communications (IZS'2000). Since 2003, he has been president and general chairman of the International Zürich Symposium on Electromagnetic Compatibility (EMC Zürich). He is a member of the Editorial Board of the IEEE TRANSACTIONS ON MICROWAVE THEORY AND TECHNIQUES. From 2000 to 2003 he was an associate editor for the IEEE MicrowAVE AND WIRELESS COMPONENTS LETTERS and from July 2003 until the end of 2005, was the editor-in chief. Since 1992, he has been on the Technical Program Committee (TPC) of the IEEE Microwave Theory and Techniques Society (IEEE MTT-S) International Microwave Symposium (IMS), IEEE MTT-S Technical Committee on Microwave Field Theory. In 1999, he was a member of the Technical Program Committee (TPC), European Microwave Conference. From 1998 to 2003, he was the chapter chairman of the IEEE Swiss Joint Chapter on Microwave Theory and Techniques (MTT), Antennas and Propagation (AP), and Electromagnetic Compatibility (EMC). He was the recipient of the 1983 Outstanding Publication Award of the Institution of Electronic and Radio Engineers, the 1996 K. J. Mitra Award of the Institution of Electronics and Telecommunication Engineers (IETE) for the best research paper in 1995, and the 2004 ACES Outstanding Paper Award. 